\title{
Self-esteem and rhinoplasty: a case-control study
}

\author{
Mohsen Naraghi ${ }^{1,2,3}$, Mohammad Atari ${ }^{4}$ \\ ${ }^{1}$ Division of Rhinology and Facial Plastic Surgery, Department of Otorhinolaryngology, Head and Neck Surgery, Tehran University of \\ Medical Sciences, Tehran 14155-6456, Iran. \\ ${ }^{2}$ Otorhinolaryngology Research Center, Tehran University of Medical Sciences, Tehran 14155-6456, Iran. \\ ${ }^{3}$ Rhinology Research Society, Tehran 14155-6456, Iran. \\ ${ }^{4}$ Department of Psychology, University of Tehran, Tehran 14155-6456, Iran.
}

Address for correspondence: Dr. Mohammad Atari, Department of Psychology, University of Tehran, Jalal-ale-Ahmad Bridge, Faculty of Psychology and Educational Sciences, Tehran 14155-6456, Iran. E-mail: atari@ut.ac.ir

\begin{abstract}
Aim: Self-esteem is one's attitude towards oneself. It is one of the most important psychological aspects of rhinoplasty, a common aesthetic operation. Prior studies have indicated an improvement in patients' self-esteem after this operation. The aim of current study was to preoperatively compare self-esteem in patients seeking aesthetic rhinoplasty with that of functional rhinoplasty patients. Methods: A total of 42 patients completed the validated Rosenberg Self-Esteem Scale preoperatively (21 aesthetic surgery patients and 21 functional surgery patients). Those with both aesthetic and functional purposes were categorized regarding their primary objective. The $t$-test for independent groups was used for analysis of the data, and Cohen's d was calculated as a measure of effect size. Results: The mean level of self-esteem in the aesthetic surgery group was significantly lower than that of the functional surgery group $(P<0.05)$. Age, gender, socio-economic status, and educational backgrounds were analyzed and comparative analysis of each showed no significant difference between the two groups. The value of effect size measure was very high $(d=1.04)$. Conclusion: The findings of the present study showed that aesthetic rhinoplasty patients had lower self-esteem in comparison with functional rhinoplasty patients.
\end{abstract}

Key words:

Cosmetic surgery; aesthetic rhinoplasty; functional rhinoplasty; self-esteem

\section{INTRODUCTION}

Beauty is admired by everyone, and facial beauty is the most important component of beauty among humans. Several studies have suggested that the perception of facial attractiveness is relatively independent of culture..$^{[1,2]}$ Attractive faces activate reward centers in the brain, ${ }^{[3]}$ motivate sexual behavior and the development of same-

\begin{tabular}{|l|l|}
\hline \multicolumn{2}{|c|}{ Access this article online } \\
\hline Quick Response Code: & Website: \\
& http://parjournal.net \\
\cline { 2 - 2 } & \\
\hline
\end{tabular}

sex alliances, ${ }^{[4,5]}$ and elicit positive treatment in various settings. ${ }^{\left[{ }^{[6]}\right.}$ As a result, it is not surprising that philosophers and scientists have long puzzled over what makes a face more attractive and why we have specific preferences. ${ }^{[7]}$

The nose plays a significant role in facial beauty. ${ }^{[8]}$ Awareness of its role in facial beauty and the emphasis on beauty by

This is an open access article distributed under the terms of the Creative Commons Attribution-NonCommercial-ShareAlike 3.0 License, which allows others to remix, tweak and build upon the work non-commercially, as long as the author is credited and the new creations are licensed under the identical terms.

For reprints contact: service@oaepublish.com

How to cite this article: Naraghi $M$, Atari $M$. Self-esteem and rhinoplasty: a case-control study. Plast Aesthet Res 2016;3:111-4.

Received: 24-09-2015; Accepted: 18-12-2015 
mass media have made aesthetic rhinoplasty one of the most frequently requested aesthetic operations worldwide. Aesthetic rhinoplasty carries tremendous potential for contouring, improving harmony, and improving the proportions of a patient's facial aesthetics, but patients occasionally demand too much of the rhinoplasty procedure.

Aesthetic plastic surgery deals with the psychosocial problems of patients in addition to their medical condition. In the past several decades, researchers have begun to explore the psychology underlying cosmetic surgeries. This aspect of cosmetic surgeries has two major aspects, the surgeons' viewpoints and the patients' points of view. The opinions of surgeons on this subject vary and have not been studied comprehensively. For patients, the strongest motivation for undergoing cosmetic surgery is body dissatisfaction, as their physical appearance constitutes an important part of their self-esteem. ${ }^{[9]}$ Body image refers to the way people perceive their bodily appearance ${ }^{[10]}$ and consists of several components. Sarwer et al. ${ }^{[11]}$ introduced a theoretical model of the association between body image and aesthetic surgery in which the two basic parts of body image, valence and body image value, play a crucial role. ${ }^{[12]}$ Body image valence refers to the importance of body image to one's self-esteem, whereas body image value describes the extent to which one is satisfied with her/his physical appearance. According to this model, those whose self-esteem highly depends on their appearance and those who have significant body dissatisfaction levels will consider cosmetic surgery.

Self-esteem is one of the psychological aspects of aesthetic surgery which has received increased attention in the past few decades. ${ }^{[13]}$ Self-esteem refers to how much people value or accept themselves for whom and what they are. It has also been defined as one's attitude towards oneself or one's opinion or evaluation of oneself, which may be positive, neutral, or negative. ${ }^{[14]}$ It has been shown that satisfaction with one's own appearance (positive body image) and self-esteem are relatively strongly correlated, especially in women. ${ }^{[15]}$

Empirical studies support the notion that aesthetic rhinoplasty patients show low levels of self-esteem. ${ }^{[16,17]}$ This study aimed to compare the mean score of self-esteem between aesthetic rhinoplasty candidates as cases and functional rhinoplasty patients as the control group.

\section{METHODS}

\section{Participants}

A total of 42 surgical patients (28 females and 14 males) were recruited for this study using the convenience sampling method from a surgical clinic in Tehran, Iran. All patients had been scheduled to undergo plastic surgery in winter 2012. Approval from the ethics committee of the Rhinology Research Society and Tehran University of Medical Sciences and informed consent were obtained. Twenty-one patients who needed functional rhinoplasty surgery were recruited as the control group while 21 cases of aesthetic rhinoplasty were included as the study group. The deformities included: dorsal hump, supratip nasal deformity, septal deviation, and dorsal irregularities. No congenital deformities were present in the cases. Secondary rhinoplasty patients were excluded given the greater risk of issues with self-esteem and body image. Those with both functional and aesthetic complaints $(n=7)$ were asked about their main objective and were categorized accordingly.

\section{Measures}

Demographics

A questionnaire consisting of information about the patient's age, gender, marital status, educational background, and subjective socio-economic status (SES) was used as the demographic questionnaire.

\section{Rosenberg Self- Esteem Scale}

Global self-esteem was measured by the Rosenberg SelfEsteem Scale ${ }^{[18]}$ which contains 10 items to be scored on a four-point Likert scale. Higher scores correspond to more positive levels of self-esteem. Scores can range between 10 and 40, with scores below 21 indicating low self-esteem. ${ }^{[19]}$ Response options were provided ranging from "strongly disagree" to "strongly agree" and were coded from 1 to 4 . Therefore, the maximum and minimum scores were 40 and 10 respectively. Cronbach's alpha in the current study was 0.86 .

\section{Procedure}

All participants from both groups completed the questionnaires preoperatively. Informed consent letters were also collected prior to administration of tests.

\section{Statistical analysis}

Data entry and analysis were performed in a blinded fashion by personnel who were not involved in the process of data collection. All tests were 1-tailed due to the hypothesis and $P$ $<0.05$ was considered statistically significant. Additionally, Levene's test was performed for assessment of equality of variances. Statistical analysis was performed using Statistical Package for Social Sciences (SPSS) software.

\section{RESULTS}

Twenty-one patients seeking aesthetic rhinoplasty and 21 patients seeking functional rhinoplasty participated in this study. The mean age in the functional rhinoplasty group was 27.48 years $(S D=10.40)$ and that of the aesthetic rhinoplasty group was 25.57 years $(S D=7.06)$. No significant difference was observed between means $(P>0.05)$. The demographic information is summarized in Table 1.

Using the $t$-test for independent groups, analysis of the data demonstrated that the mean self-esteem score in the aesthetic surgery group was significantly lower than that of the functional surgery group $(P<0.01)$. Age, gender, SES, and educational backgrounds were analyzed and no significant differences were found between the two groups $(P>0.05)$. Moreover, the Levene's test for equality of variances was not significant $(P>0.05)$. Independent $t$-test details are presented in Table 2. 
Table 1. Demographic characteristics of cases and controls

\begin{tabular}{lll}
\hline Characteristics & Aesthetic rhinoplasty patients & Functional rhinoplasty patients \\
\hline $\mathrm{n}(\%)$ & $21(50 \%)$ & $21(50 \%)$ \\
Mean age & 25.27 & 27.48 \\
Gender & 5 & 9 \\
$\quad$ Male & 16 & 12 \\
$\quad$ Female & 11 & 14 \\
Marital status & 9 & 6 \\
$\quad$ Single & 1 & 1 \\
Married & 9 & \\
Separated & 11 & 8 \\
Educational level & 1 & 12 \\
Low & & 1 \\
Medium & 2 & 3 \\
High & 15 & 15 \\
Socio-Economic Status & 4 & 3 \\
Low & & \\
Medium & & \\
High & & \\
\hline
\end{tabular}

Table 2. Independent $t$-test details between aesthetic and functional groups

\begin{tabular}{llllc}
\hline Group & Mean & SD & t-test statistic $\boldsymbol{P}$ value & Cohen's d \\
\hline Aesthetic & 29.667 & 3.954 & 3.911 & $P<0.011 .04$ \\
Functional & 34.095 & 3.360 & & \\
\hline
\end{tabular}

\section{DISCUSSION}

The objective of this study was to compare self-esteem between candidates applying for aesthetic rhinoplasty and patients seeking functional nasal surgery. Both groups were evaluated for other variables including age, gender, SES, and education, and no significant differences were found.

Findings from statistical analyses suggest that cosmetic rhinoplasty candidates have lower levels of self-esteem in comparison with functional patients. Results are consistent with prior studies suggesting that aesthetic rhinoplasty patients have lower scores in self-esteem and body-image measures. ${ }^{[16,17]}$ It has also been reported that women with higher self-esteem have less interest in cosmetic surgery, and that a negative body-image is associated with lower self-esteem. ${ }^{[9]}$ Results of the present study are in line with these findings. The magnitude of effect size in the present study shows that there is a clinically meaningful difference in self-esteem between aesthetic rhinoplasty patients and functional rhinoplasty patients.

The findings of the present study are limited to behavioral manifestations prior to surgery. Other studies have evaluated the long-term outcomes of cosmetic surgery, and have demonstrated that aesthetic surgery can statistically improve self-esteem, depression, and anxiety. ${ }^{[20]}$ However, only a small effect size $(d=0.15)$ has been reported for an improvement in self-esteem prior to and 6 months after aesthetic surgery. ${ }^{[21]}$ Other psychological variables including depression and anxiety have also shown improvement following aesthetic surgery. ${ }^{[2]}$ The findings of the current study are consistent with another study which reported high scores of depression among aesthetic rhinoplasty patients. ${ }^{[23,24]}$ This study also supports the notion that an interest in aesthetic rhinoplasty is associated with a negative body image. ${ }^{[25]}$

The present study shows that aesthetic rhinoplasty patients are more likely to have low levels of self-esteem. Some limitations of the study are worth noting. First, the utilized instrument could have been more comprehensive by including more aspects of self-esteem. Second, two patients in the aesthetic group showed extremely low levels of self-esteem, which lowered this group's mean score. It is recommended for future research to evaluate the differences using various psychometric instruments by more accurate sampling methods.

In conclusion, the current findings indicate that evaluation of the psychological condition of aesthetic rhinoplasty candidates is of great value. Therefore, cosmetic surgeons might screen patients with psychometric instruments ${ }^{[26]}$ and/or consider cooperating with psychologists. Moreover, cosmetic surgeons can gain skills to recognize potential psychologically problematic patients. In this respect, it is crucial for cosmetic surgeons to be familiar with the psychology of cosmetic surgery and related disorders.

\section{Financial support and sponsorship Nil.}

\section{Conflicts of interest}

There are no conflicts of interest.

\section{REFERENCES}

I. Cunningham MR, Roberts AR, Barbee AP, Druen PB, Wu CH. Their ideas of beauty are, on the whole, the same as ours: consistency and variability in the crosscultural perception of female physical attractiveness. J Personal Soc Psychol 1995;68:26I-79.

2. Langlois JH, Kalakanis L, Rubenstein AJ, Larson A, Hallam M, Smoot M. Maxims or myths of beauty? A meta-analytic and theoretical review. Psychol Bull 2000; I 26:390-423.

3. Aharon I, Etcoff NL, Ariely D, Chabris CF, O'Connor E, Breiter HC. Beautiful faces have variable reward value: $\mathrm{MRI}$ and behavioral evidence. 
Neuron 200I;32:537-5I.

4. Berscheid E, Reis HT. Attraction and close relationships. In: Gilbert DT, Fiske ST, Lindzey G, editors. The Handbook of Social Psychology. 4th ed. Oxford: Oxford University Press; 1998. p. 193-281.

5. Rhodes G, Simmons L, Peters M.Attractiveness and sexual behaviour: does attractiveness enhance mating success? Evol Hum Behav 2005;26: I86-20I.

6. Hosoda M,Stone-Romero EF, Coats G.The effects of physical attractiveness on job related outcomes: a meta-analysis of experimental studies. Pers Psychol 2003;56:43I-62.

7. Etcoff N. Survival of the Prettiest: The Science of Beauty. New York: Anchor/Doubleday; 1999.

8. Amodeo CA. The central role of the nose in the face and the psyche: review of the nose and the psyche. Aesthet Plast Surg 2007;31:406-10.

9. Sarwer DB, Wadden TA, Pertschuk MJ, Whitaker LA. The psychology of cosmetic surgery: a review and conceptualization. Clin Psychol Rev 1998; 18:1-22.

10. Cash TF. Body image: past, present, and future. Body Image 2004; I:I-5.

II. Sarwer DB, Whitaker LA, Pertschuk MJ,Wadden TA. Body image concerns of reconstructive surgery patients: an underrecognized problem. Ann Plast Surg 1998;40:403-7.

12. Cash TF, Pruzinsky T. Body image: a handbook of theory, research and clinical practice. New York, NY:The Guilford Press; 2002.

13. Figueroa $C$. Self-esteem and cosmetic surgery: is there a relationship between the two? Plast Surg Nurs 2003;23:21-4.

14. Colman AM. Oxford dictionary of psychology. Oxford: Oxford University Press; 2009.

15. Feingold A. Good-looking people are not what we think. Psychol Bull |992; | | |:304-4|.
16. Phillips KA, Pinto A, Jain S. Self-esteem in body dysmorphic disorder. Body Image 2004; I:385-90.

17. Pecorari G, Gramaglia C, Garzaro M,Abbate-Daga G, Cavallo GP, Giordano C, Fassino S. Self-esteem and personality in subjects with and without body dysmorphic disorder traits undergoing cosmetic rhinoplasty: preliminary data. J Plast Reconstr Aesthet Surg 2010;63:493-8.

18. Rosenberg M. Society and the adolescent self-image. Princeton, NJ: Princeton University Press; 1965.

19. Rosenberg M. Conceiving the self. New York: Basic Books, Inc.; 1979.

20. Moss TP, Harris DL. Psychological change after aesthetic plastic surgery: a prospective controlled outcome study. Psychol Health Med 2009; | 4:567-72.

21. Von Soest T, Kvalem IL, Roald HE, Skolleborg KC. The effects of cosmetic surgery on body image, self-esteem, and psychological problems. J Plast Reconstr Aesthet Surg 2009;62: I238-44.

22. Sarwer DB, Gibbons LM, Magee L, Baker JL, Casas LA, Glat PM, Young VL. A prospective, multi-site investigation of patient satisfaction and psychosocia status following cosmetic surgery. Aesthet Surg J 2005;25:263-9.

23. Naraghi M, Atari M. Comparison of patterns of psychopathology in aesthetic rhinoplasty patients versus functional rhinoplasty patients. Otolaryngol Head Neck Surg 20 I5; I52:244-9.

24. Naraghi M,Atari M.A comparison of depression scores between aesthetic and functional rhinoplasty patients. Asian J Psychiatr 20I 5; 14:28-30.

25. Atari M, Akbari-Zardkhaneh S, Mohammadi L, Soufiabadi M. The factor structure and psychometric properties of the Persian version of body appreciation scale. Am J Appl Psychol 20I5;3:62-6.

26. Wildgoose P, Scott A, Pusic AL, Cano S, Klassen AF. Psychological screening measures for cosmetic plastic surgery patients: a systematic review. Aesthet Surg J 2013;33:152-9. 